ably on account of absorption in the cloud regions of our atmosphere, which, as Langley has shown, takes up with great avidity the violet and ultra-violet rays.

May it not be that in clouds we have conditions especially favourable to the production of the Hertz effect? If so, the discharge from one cloud to another would be accompanied by in earth current in the opposite direction, as in the theory proposed by Prof. Stokes, in which a decrease of resistance is produced by an increase of heat from the sun.

Hertz found his effect (Wied. Ann., xxxi. p. 993) much more marked in a medium under diminished pressure.

Under 300 millimetres of mercury, he finds that the ultraviolet radiation will nearly quadruple the length of spark obtained without it, while under ordinary atmospheric pressure it would scarcely double it. But this is the very circumstance which is realized in the case of cl uds.

There is also reason to think that solar outbursts are especially rich in these rays of short wave-leng!h which are required to explain the phenomena.

Haverford College, U.S.A., March 22.

\section{Hertz's Equations in the Field of a Rectilinear Vibrator.}

RECURRING to Herts's equations for the field of the rectilinear vibrator, it appears to me that, while his conclusions are sound as regards the forces at points very distant from the vibrator, they require modification for the rest of the field. In fact, the principles upon which the question is investigated require that the electromotive force in the direction of $z$ should become evanescent close to the vibrator (the axis of $z$ ).

The general form of $\Pi$ is either-

$$
\frac{\mathrm{M} \sin \frac{\rho}{\lambda}}{\rho} \cdot \sin n t \text {, or } \frac{\mathrm{M} \cos \frac{\rho}{\lambda}}{\rho} \cdot \sin n t,
$$

where $\lambda$ large, and $\lambda n=\frac{\mathrm{I}}{\mathrm{A}}$, or, of course, the sum of the two forms.

In assuming for points near the origin (say the middle point of the vibrator) the approximate expression--

$$
\frac{\mathrm{M}}{\rho} \sin n t,
$$

Hertz, in point of fact, takes the second of the above forms for $\Pi$, for this reduces to $\frac{M}{\lambda} \cdot \sin n t$ when $\frac{\rho}{\lambda}$ is very small.

But this assumption makes both $\Pi$ and $\mathrm{Z}$ infinitely great close to the vibrator. Whereas, by assuming the former of the two forms, or-

$$
\frac{M \sin \frac{\rho}{\lambda}}{\rho} \cdot \sin n t,
$$

i.e. near the origin $\Pi=\frac{M}{\lambda} \sin n t$, we get, as a general expression for $\mathrm{Z}$ -

$Z=M\left\{\left(\frac{\mathrm{I}}{\rho^{3}}-\frac{3 z^{2}}{\rho^{5}}+\frac{z^{2}}{\lambda^{2} \rho^{3}}-\frac{\mathrm{I}}{\lambda^{2} \rho}\right) \sin \frac{\rho}{\lambda}-\left(\frac{\mathrm{r}}{\lambda \rho^{2}}-\frac{3^{2}}{\lambda \rho^{4}}\right) \cos \frac{\rho}{\lambda}\right\} \sin n t$, and, as $\rho$ is indefinitely diminished, this reduces to-

as a limiting value.

$$
-\frac{2}{3} \frac{\mathrm{M}}{\lambda^{3}} \sin n t
$$

For distant portions of the field, Hertz's results as to the laws and amplitudes of the forces electric and magnetic remain unaltered.

Of course, the whole investigation, with such a simple assumption as to the nature of the field, must be regarded as only approximate. For any given form of vibrator-as, for example, a straight wire connecting two spheres-the exact treatment will be very difficult. In the simplest conceivable case of a spherical metal sheet with an induced $Q_{n}$ distribution l. $\mathrm{ft}$ to itself, the analysis is intricate (see a paper by Prof. J. J. Thomson to the Mathematical Society of London, January I884).

Berkswell, March 29.

\section{Early History of Lightning-Conductors.}

CAN any of your readers refer me to the sources of some of the late Mr. Richard Anderson's information with regard to the early history of the lightning-conductor? (I) On p. 27 of the third edition (1885) of his book, "Lightning.Conductors,". he states that Franklin, in the 1758 issue of "Poor Richard," gave directions for the erec:ion of lightning-conductors. (2) On p. 25 he refers to Prof. Winthorp, of Boston, having, in 1755, defended the lightning-conductor against a parson who had attributed a Massachusetts earthquake to the innovation. I shou'd be much obliged for any reference to a library where a copy of "Poor Richard" for 1758 could be found ; or, again, for any information with regard to Winthorp's defence of the lightning-conductor.

Prof. Meidinger, of Karlsruhe, who is preparing a second edition of his "History of Lightning-Conductors," is extremely desirous of verifying these details of their early history, and I should be glad if any of your readers could supply me with information for him on these points. KARL PEARSON.

University College, April 9.

\section{The Satellite of Procyon.}

Mr. J. M. Barr's suggestion (NATURe, March 28, p. 5 ro), as to the use of photography to ascertain whether there is any close companion or satellite to Procyon, would be considered a very desirable one by astronomers, in order to set at rest the question whether a companion can actually be disc svered near the assumed place of the hypothetical one, of which the elements were given by Dr. Auwers in $186 \mathrm{r}$, from investigations of the irregularity in the proper motion of Procyon observed by Bessel in 1844 , and by Mädler in $185 \mathrm{I}$. The orbit was computed on the assumption of a circular motion in a plane perpendicular to the line of sight round a point about $\mathbf{I}^{\prime \prime} \cdot 2$ distant, having a period of about 40 years, the position angle for 1873 being about $90^{\circ}$, so that the present angle would be about $234^{\circ}$, or about $9^{\circ}$ per annum.

I fear, however, serious instrumental difficulties would arise in obscuring such a brilliant object as Procyon in a large telescope by a screen, so as to get the impress on a plate of a probably faint companion at the extremely close distance of two to three seconds of arc.

This difficulty, no doubt, must have presented itself to the minds of the astronomers at the Lick Observator $y$, California, or they would have tried the sensitive plate with the 34 -inch photo lens of the great refractor, instead of examining Procyon visually with the 36 -inch glass, as was clone by Mr. S. W. Burnham on the early morning of November 18 last, with the following record:- "Procyon. - Carefully examined with all powers up to 3300 on the 36 -inch under favourable conditions. Large star single, and no near companion."

If this means that no companion was seen within $\mathbf{I O}^{\prime \prime}$ or $\mathbf{1 2}^{\prime \prime}$ radius, it makes the matter very perplexing, as Otto Struve measured a supposed new companion in 1873 with the 15 -inch refractor at Pulkowa-the mean of several measures for March 28 being $P$. angle $90^{\circ} \cdot 24$, and distance $12^{\prime \prime} \cdot 49$, and for 1874 (April IO) P. angle $99^{\circ} \cdot 6$, and distance $\mathrm{II}^{\circ} \cdot 67$. This companion was looked for at Washington with the 26 -inch refractor on several occasions from November 1873 till January 1876 , and by the three Clarks (father and two sons) with the McCormick 26-inch refractor, then completed at Cambridgeport, Massachusetts, but Struve's companion could not be seen with either instrument, and I am not aware that it has since been seen by Struve himself with the new Russian 30-inch refractor. The Washington observers at that time, however, gave estimated places for three new companions, supposed to be seen by them as follows :-

No. I. Position angle, about $10^{\circ}$, and distance about $6^{\prime \prime}$.

,, 2. ", ," $, 36^{\circ},, \quad, \quad, \quad 8^{\prime \prime} \cdot 8$

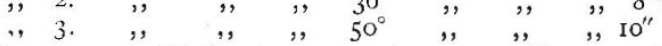

These appear (if they have an existence at all) to have been missed with the 36 -inch glass at the Lick Observatory, as above referred to.

It is a singular coincidence that the position-angles of the companion supposed to have been seen by Otto Struve in 1873 and 1874 agreed with the orbital places computed by Dr. Auwers, but its distance involved the assumption of an enormous mass to Procyon for the parallax assigned to the principal star.

Belfast, April r.
ISAAC W. WARD. 